\title{
O Terapeuta Ocupacional e o Cuidador na construção de material lúdico em uma
}

\section{Enfermaria Pediátrica}

The Occupational Therapist and the Caregiver in the fabrication of playful material in a Pediatric

Ward

EI Terapeuta Ocupacional y el Cuidador em la construcción de material lúdico em una Enfermería Pediátrica

Sandy de Oliveira Lemos Gomes ORCID: https://orcid.org/0000-0003-3152-5152 Unimed Recife, Brasil E-mail: terapeutaocuacional.sandy@gmail.com

Anna Paula Aca Ferreira ORCID: https://orcid.org/0000-0002-4666-1635 Instituto de Medicina Integral Professor Fernando Figueira, Brasil E-mail: Annapaula_aca@hotmail.com

Marcela de Paula Conceição de Andrade Oliveira ORCID: https://orcid.org/0000-0002-0699-1779 Centro Especializado em Apoio Multidisciplinar, Brasil E-mail: marcelaandrade_2011@hotmail.com

Ana Carollyne Dantas de Lima ORCID: https://orcid.org/0000-0002-2735-4090 Universidade Federal da Paraíba, Brasil E-mail: ana_cdlima@yahoo.com.br

Kelly Lins Serafim

ORCID: https://orcid.org/0000-0001-7141-8680 Consultório Privado, Brasil E-mail: kellylinsserafim@gmail.com

Ana Carolina Cavalcanti Silva Santos ORCID: https://orcid.org/0000-0002-8325-385X Consultório Privado, Brasil E-mail: carolzcavalcnti@gmail.com

Aneide Rocha de Marcos Rabelo

ORCID: https://orcid.org/0000-0001-6492-821X Universidade Federal de Pernambuco, Brasil E-mail: rabelo.ufpe@gmail.com

\begin{abstract}
Resumo
Objetivo: Este estudo tem como objetivo apresentar como a humanização a partir do oferecimento e construção de um brinquedo pode mudar o cotidiano da enfermaria. Metodologia: tratar de um estudo descritivo de abordagem qualitativa. A pesquisa foi desenvolvida a partir da leitura dos diários de campo dos encontros realizados com 68 cuidadores, de forma grupal e individual, nas enfermarias, para a construção de material lúdico, o qual faziam parte Chocalho de material reciclado e Placa de Identificação de EVA. Resultados: Como resultado, tem-se a apresentação da proposta, a construção do material, as orientações e a devolutiva dos participantes. Discursão: A partir da intervenção, o facilitador, os cuidadores e a equipe relataram como a enfermaria ficou mais humanizada, modificando o cotidiano, facilitando o diálogo e diminuindo estresse do cuidador e sua criança. Conclusão: Conclui-se que mais ações nas enfermarias são necessárias, pois com adesão de novas técnicas nesses ambientes pode ser alcançado um maior bem estar para o cuidador e a criança.
\end{abstract}

Palavras-chave: Cuidador; Enfermarias; Jogos e brinquedos; Terapia ocupacional.

\section{Abstract}

Objective: This study aims to present how humanization from the offer and fabrication of a toy can change the daily life of the ward. Methodology: This is a descriptive study, with a qualitative approach. The research was developed in the wards, from reading the field diaries of the meetings held with 68 caregivers, in a group and individually, for the construction of playful material, rattle and EVA Identification Plate, which were part of recycled material. Results: As a result, we have the presentation of the proposal, the construction of the material, the guidelines and feedback from the 
participants. Discussion: From the intervention, the facilitator, the caregivers and the team reported how the ward became more humanized, changing the daily routine, facilitating the dialogue and reducing the stress of the caregiver and their child. Conclusion: It is concluded that more actions in the wards are necessary, once the acceptance of new techniques in these environments, greater well-being can be achieved for the caregiver and the child.

Keywords: Caregivers; Wards; Play and toys; Occupational therapy.

\section{Resumen}

Objetivo: Este estudio tiene como objetivo presentar cómo la humanización a partir de la oferta y construcción de un juguete puede cambiar la vida diaria de la sala. Metodología: Se trata de un estudio documental, con enfoque cualitativo. La investigación se desarrolló a partir de la lectura de los diarios de campo de las reuniones realizadas con 68 cuidadores, de forma grupal e individual, en las salas, para la construcción de material lúdico, que formaron parte de un sonajero de material reciclado y Placa de Identificación EVA. Resultados: Como resultado, tenemos la presentación de la propuesta, la construcción del material, los lineamientos y la retroalimentación de los participantes. Discurso: A partir de la intervención, el facilitador, los cuidadores y el equipo informaron cómo la sala se humanizó más, cambiando la rutina diaria, facilitando el diálogo y reduciendo el estrés del cuidador y su hijo. Conclusión: Se concluye que son necesarias más acciones en las salas, pues con la adherencia de nuevas técnicas en estos ambientes se puede lograr un mayor bienestar para el cuidador y el niño.

Palabras clave: Cuidadores; Habitaciones de pacientes; Juego e implementos de juego; Terapia ocupacional.

\section{Introduçãa}

A criança ao adoecer, muitas vezes, poderá necessitar de um cuidado mais especializado acarretando a hospitalização. Durante o internamento conta-se com a ajuda de um cuidador, o qual em sua maioria não receberá remuneração, sendo este denominado de cuidador informal. Dentre os cuidadores informais, vemos que em sua maioria são compostos por familiares das crianças, como, mães, pais e avós (Pylo, Peixoto \& Bueno, 2015; Chesani, Andrade, Kerkoski, 2019).

A partir da inserção da criança e de seu cuidador no ambiente hospitalar vê-se ser desencadeado estresse em ambos diante das novas regras a que estão sujeitos, bem como, as limitações do espaço e a divisão deste com pessoas desconhecidas. Outro fator que influência negativamente no bem estar do cuidador é a maior dependência apresentada pela criança em suas atividades de vida diária, por causa da enfermidade e das condições ambientais (Bustamante, Neves, Matos \& Oliveira, 2014).

Sabe-se que o apoio dos profissionais que os cercam produzirá a sensação de uma maior segurança diante de toda essa vivência. O suporte ofertado pela equipe hospitalar que o acompanha diariamente, apresentando atenção voltada ao cuidador, poderá evitar muitos problemas secundários (Chesani, Andrade \& Kerkpski, 2019). Dentro dessa ótica o terapeuta ocupacional tem como papel em sua atuação na enfermaria utilizar de seus saberes técnicos para criar um ambiente mais humanizado, fazendo uso de atividades para promover o cuidado, e a interação entre cuidador-criança-equipe hospitalar, favorecendo o bem estar dos envolvidos (Rodrigues \& Albuquerque, 2020).

Salienta-se que para a humanização na enfermaria pediátrica, pode ser visto a partir da introdução de brinquedos no leito, necessários para amenizar a situação vivenciada pela criança, lhe trazendo conforto, assim como, a orientação dos cuidadores quanto a importância desse brinquedo para o desenvolvimento da criança durante o período de internamento. Dessa maneira, os cuidadores também demonstram maior tranquilidade, quando a criança se apresenta estável (Bustamente, et. al, 2014).

O brincar é uma atividade natural da criança, que constitui parte da sua aprendizagem e a faz desenvolver fisicamente e psicologicamente. Na enfermaria está atividade apresenta-se de forma limitada, porém, o brinquedo nesse processo se faz de suma importância. Em geral, este será introduzido pelos pais, para acalmar as crianças e fazer do ambiente hospitalar um local mais acolhedor, por fim estará auxiliando na aprendizagem de novas habilidades da criança (Silva, Ballarin \& Oliveira, 2015; Brasil, 2017; Souza, Figueiredo, Silva, 2017; Ribeiro, 2020).

Assim, a fim de apresentar como a humanização, a partir do oferecimento de brinquedos no ambiente hospitalar pode influenciar na rotina dos cuidadores e crianças, será observado nesse estudo o envolvimento dos cuidadores na construção destes materiais lúdicos para as crianças. 


\section{Metodologia}

Trata-se de um estudo documental, de abordagem qualitativa. Os dados da pesquisa documental são coletados a partir de fontes não bibliográficas. Nessas pesquisas se usam fontes como cartas pessoais, gravações, memorandos, diários de campo (Menezes, Duarte, Carvalho \& Souza, 2019). A abordagem qualitativa apresenta-se em um meio natural e com a coleta direta pelo pesquisador, são dados descritos, visando o produto e sua relação com as pessoas, que são o centro de atenção para o pesquisador. A análise de dados se dá através de um processo indutivo (Pereira, Shitsuka, Parreira \& Shitsuka, 2018). O estudo foi desenvolvido nas enfermarias de um Hospital Escola localizado em uma capital Nordestina, cada enfermaria era composta por 8 leitos.

Como critério de inclusão foram incluídos os cuidadores que acompanhavam crianças nas enfermarias de D à H, e como critério de exclusão, os cuidadores que estavam nas enfermarias de A à C, por estes serem leitos de Unidade de Terapia Intensiva Neonatal. Foram realizadas 15 intervenções grupais e 17 individuais, das quais tiveram 68 participantes. Para manter o sigilo sobre o nome dos participantes, pesquisadores e equipe que contribui com os relatos para os diários de campo, os cuidadores foram identificados pela letra C (Letra para cuidador), o facilitador pela letra F (Letra inicial da palavra facilitador) e seguido pelo número no qual o participante aparece no Diário de Campo (C1, C2, C3... F1, F2...). Enquanto para identificar a equipe do setor, foi utilizado Equipe Hospitalar.

Para a coleta de dados foram utilizados os diários de campo redigidos pelas pesquisadoras, os quais continham informações como: número e caracterização dos encontros na enfermaria na qual foi realizada a ação, identificação do material lúdico produzido, além da descrição da oficina, considerando as facilidades e dificuldades relatadas durante a vivência. As intervenções foram realizadas nas formas grupais e individuais, nos leitos da enfermaria pediátrica em um único momento, com duração média de 40 minutos.

A intervenção com o cuidador aconteceu da seguinte forma: inicialmente foi explicado quem era o terapeuta ocupacional e qual o seu papel na enfermaria e apresentando o material que poderia ser confeccionado. Considerando as características da enfermaria, o quadro clínico da criança, optou-se pela construção de placas de identificação e de chocalhos, na perspectiva de facilitar a interação cuidador-criança, promover o lúdico e prevenir danos para ambos.

O material foi produzido de forma ativa e dialogada, sendo ainda fornecido a este cuidador informações acerca do desenvolvimento infantil, tendo como base a Caderneta da Criança, bem como orientações de como utilizar o brinquedo de forma individualizada. Foi ressaltada ainda a relevância do adulto quanto ao acesso à exploração do ambiente e dos brinquedos em geral, pela criança.

Ao final da ação, havia a escuta e discussão da devolutiva dos cuidadores e da equipe hospitalar, para realização do registro de falas e observações nos Diários de Campo. Após análise do conteúdo, foi identificado nos relatos às categorias: melhora das relações interpessoais, humanização, quebra do ócio e envolvimento dos cuidadores na atividade (Minayo, Deslandes \& Gomes, 2011).

Quanto ao material para a confecção das placas de identificação, foram colocados à disposição dos cuidadores alguns moldes em EVA (coração, galinha pintadinha, peixe, flor e borboleta), palitos de picolé e cola de silicone. O cuidador escolhia o molde e a cor da placa, recortava-os, fazia a colagem e na parte de trás colocava o palito. Este material poderia ser fixado no berço da criança, funcionando também como identificação da criança. Para o chocalho foram utilizadas garrafas pet de $250 \mathrm{ml}$, canudos, lantejoulas e pedaços de emborrachado (EVA) de cores diversos picados pelo cuidador. A garrafa era preenchida até um terço e logo em seguida era utilizada a fita adesiva colorida para vedar a tampa, sendo o material identificado com o nome da criança. Enfatizada também a importância da utilização de contraste para a construção do material lúdico.

Vale salientar que alguns cuidados para prevenção de infecção hospitalar foram tomados durante o preparo da atividade e a confecção / utilização do material lúdico. As garrafas pet foram previamente higienizadas, além disso, os materiais foram 
distribuídos aos cuidadores em sacos plásticos para contribuir com a manutenção da organização do ambiente e guarda de todo o resíduo produzido. As etapas eram dadas passo a passo, respeitando o ritmo e a necessidade de cada cuidador.

\section{Resultados}

Tem-se um total de 68 participantes, distribuídos em 15 intervenções grupais e 17 individuais. Destes 96\% eram do gênero feminino e $4 \%$ do gênero masculino. A idade média dos cuidadores foi de 36 anos, com a idade mínima de 18 e a máxima de 60 anos.

No que se refere ao processo de construção do material lúdico, foram consideradas quatro etapas apresentadas no Quadro 1.

Quadro 1: Identificação dos principais pontos relacionados ao processo de construção do material lúdico. IMIP, Recife/PE, 2015.

\begin{tabular}{|ll|}
\hline \multicolumn{1}{|c|}{ Etapas } & \multicolumn{1}{c|}{ Pontos identificados } \\
\hline Apresentação da proposta & Diálogo aberto, participação ativa, humanização do espaço. \\
Construção do material & Respeito ao ritmo e necessidade de cada um, passo a passo, \\
& conhecimento prévio. \\
Informação e orientação & Uso da caderneta da criança, espaço para questionamento, \\
& esclarecimento quanto à função do brinquedo e do brincar. \\
Devolutiva & Humanização, melhora do vínculo cuidador-criança, participação \\
& ativa dos cuidadores, ajuda a passar o tempo. \\
\hline
\end{tabular}

Fonte: Diário de Campo.

Outros aspectos avaliados no processo de construção do material lúdico foram as facilidades e dificuldades encontradas e relatadas pelos envolvidos, listadas no Quadro 2, a seguir.

Quadro 2: Apresentação das facilidades e dificuldades durante o processo de construção do material lúdico. IMIP, Recife/PE, 2015

\begin{tabular}{|ll|}
\hline \multicolumn{1}{|c|}{ Facilidades } & \multicolumn{1}{c|}{ Dificuldades } \\
\hline Valorização do trabalho pela equipe & Não entendimento da função do brinquedo \\
Material utilizado de baixo custo e conhecido & Desgaste do cuidador pelo processo de \\
dos cuidadores & hospitalização \\
Afinidade com trabalhos manuais & Quadro clínico das crianças exigindo um maior \\
& suporte ao cuidador \\
& Rotina / procedimentos da enfermaria \\
\hline
\end{tabular}

Fonte: Diário de Campo.

\section{Discussão}

Durante a coleta de dados, acessada por meio dos Diários de Campo, pode ser constatada a presença de apenas três homens como acompanhantes das crianças internadas. Esse dado é semelhante aos da literatura que indicam, em sua maioria, 
que os cuidadores são do sexo feminino, o que pode ser justificado pelo fato que o cuidado ser atribuído como uma função feminina na sociedade, além do instinto materno que direciona as mulheres a abandonarem suas demais funções e cuidarem dos filhos de forma integral. Também e observada uma maior sobrecarga sobre as cuidadoras (Pereira, Matsue, Vieira, Pereira, 2014; West, Vasconcelos, Coriolano-Marinus, Araújo, 2020; Pinheiro et al., 2021).

Considerando as especificidades desse público, a dinâmica das enfermarias e o relato de outros estudos, foram feitas aos cuidadores propostas de intervenções de forma grupal e individual. A proposta em grupo vem facilitar a relação interpessoal e a diminuição do estresse, tornando o ambiente favorável à troca de experiências e a questionamentos diversos (Bustamente et al., 2014; Dahdah, Carvalho, Delsim, Gomes, Miguel, 2013). Já os atendimentos individuais permitem situações específicas de sensibilização, na qual a particularidade será o foco de atenção, permeando entre aspectos técnicos e privativos, singular a cada sujeito (Moura, Guimarães, Luz, 2013). Em sequência apresenta-se o relato.

A cuidadora estava um pouco chorosa inicialmente. Depois da intervenção individual com a terapeuta ocupacional, ficou mais motivada em participar do grupo, fazendo a placa de forma criativa. (F1)

A prática na enfermaria pediátrica propiciou maior adesão dos participantes para a construção do material lúdico, facilitando a troca de informações. A apresentação da proposta realizada por meio de um diálogo aberto, respeitando as necessidades do cuidador, a humanização do espaço e a saúde dos participantes foi ao encontro as fontes pesquisadas (Joaquim, et al., 2014; Silva, et al., 2015; Chesani, Andrade \& Kerkpski, 2019).

Tia receptiva e criativa. A ela (a tia) foi mostrado como usar o recurso (placa de identificação) para estimular a criança. $(F 1)$

A construção do material lúdico teve como premissa básica a participação ativa do cuidador, respeitando o seu ritmo e o seu conhecimento prévio acerca da atividade realizada, explicando o passo a passo de forma individualizada. Em Silva et al. (2015), nota-se que em algumas dinâmicas grupais aplicadas, alguns cuidadores demandaram maior atenção que os demais, seja por meio das suas falas, como também por alegarem não possuir habilidades necessárias para a confecção de algum material específico.

Eu não sei desenhar, sou péssima nisso, mas vou fazer com todo carinho para meu bebê. (C 10)

Após a confecção do material lúdico foram compartilhadas informações sobre o desenvolvimento infantil, o uso da Caderneta da Criança e orientações sobre brinquedos e brincadeiras típicas à idade. Optou-se por utilizar a Caderneta da Criança por ela ser preconizada pelo Ministério da Saúde e conter dados referentes aos principais marcos do desenvolvimento, servindo também como guia de acompanhamento para os pais (Brasil, 2020).

\section{A mãe relatou já conhecer e acompanhar o desenvolvimento do seu filho pela Caderneta da Criança. $(F 1)$}

O papel do brincar e das brincadeiras no desenvolvimento infantil foi outro foco da intervenção realizada na enfermaria pediátrica. Bustamante et al. (2014), afirmam em seu estudo, que para a criança o brincar é uma forma de aprendizado, de aproximação dos demais, e os pais tem papel fundamental nesse processo, pois são eles que oferecem os primeiros brinquedos aos seus filhos. 
Não tinha me dado conta de como isso (brinquedo) é importante para o meu bebê. E nem precisa ser caro. (C 24)

É até bom um brinquedo, porque ela está estressada, apesar de que trouxe a boneca. (C 15)

Durante a leitura dos Diários de Campo percebe-se que a atenção dada ao cuidador durante a produção do material lúdico funcionou como um diferencial à sua saúde. Segundo Sposito et al, (2018), o contexto hospitalar pode ser um local passível a traumas, e é no brincar que os profissionais tendem a aproximar-se de forma mais amistosa das crianças internadas e dos seus cuidadores. A presença do cuidador junto à criança torna o ambiente hospitalar menos hostil e ajuda na formação de vínculos com a equipe que auxilia no tratamento (Chesani, Andrade \& Kerkpski, 2019). Em contrapartida, no estudo realizado por Dahdah et al. (2013) pode ser observado que mesmo sabendo da importância do cuidador no espaço hospitalar, a equipe de saúde não se responsabiliza por um cuidado integral junto ao mesmo.

Vocês estarem aqui foi muito importante para nós, estávamos tristes, íamos chamar à psicóloga, voltem mais vezes. (C 42)

Durante a intervenção descrita, percebe-se uma preocupação com a linguagem utilizada, sendo esta clara, objetiva e de fácil compreensão, de acordo com as habilidades de cada cuidador, facilitando a interação com os facilitadores. Chesani, Andrade \& Kerkpski, (2019) afirmam que uma comunicação muito técnica entre o profissional de saúde e o cuidador, dificulta a compreensão do mesmo, construindo uma barreira entre ambos.

A quebra do ócio também foi um fator observado durante esta pesquisa. Para Silva et al. (2015), a criação de espaços de atendimento ao acompanhante pode lhe dar qualidade de vida, já que pouco ele é lembrado durante a internação de suas crianças.

Ajudou a ocupar nosso tempo. (C 6)

Ao final da confecção mostram-se satisfeitas com o que foi realizado. (F2)

O envolvimento dos cuidadores nos grupos e na construção do material, favorece um ambiente mais humanizado e agradável, a diminuição das tensões, o fortalecimento dos vínculos entre cuidadores e crianças, pontos também reforçados por Bustamante, et al., 2014.

$O$ ambiente ficou mais alegre. (C51)

Foi muito bom vocês aqui a gente ficou mais ativas, e mais felizes. (C46)

Os cuidadores/familiares percebem que durante a visita dos profissionais de saúde, conseguem acompanhar a melhora do estado de saúde das crianças enfermas e veem o empenho dos profissionais da saúde durante a internação. (Chesani, Andrade \& Kerkpski, 2019)

Esse trabalho que vocês fazem aqui na enfermaria pediátrica é muito importante, traz humanização a esse lugar. (Equipe Hospitalar)

A atuação do terapeuta ocupacional na enfermaria pediátrica se colocou na busca pela humanização, respeito ao cuidador, inserção deste no cuidado, vendo-o como um sujeito que precisa também de atenção. Outros estudos indicam ainda 
que o terapeuta ocupacional ajuda na organização da rotina dos cuidadores, para diminuir a sobrecarga deste e favorece o setting para que esse cuidador se sinta acolhido para trazer suas angústias, encontrar apoio e chegar às soluções. (Dahdah et al., 2013; Rodrigues \& Albuquerque, 2020).

\section{Parece que a atividade transporta as cuidadoras daquele ambiente. $(F 2)$}

Dessa forma, faz-se necessário o desenvolvimento de novos estudos e de ações direcionadas especificamente ao cuidador, de forma que ele possa não apenas cuidar da sua criança, mas também de si próprio. Para tal, o trabalho interdisciplinar e a postura ativa de todos os envolvidos são essenciais nesse processo de humanização, minimizando os efeitos da hospitalização.

\section{Conclusão}

Percebe-se que apesar do reconhecimento da importância da presença do cuidador durante a hospitalização infantil, o suporte que ele recebe da equipe é mínimo ou inexistente. A partir dessas constatações o terapeuta ocupacional em sua atuação na enfermaria pediátrica utilizou-se da confecção direta pelo cuidador de materiais lúdicos para suas crianças.

As ações realizadas com esse público foram relevantes para a diminuição do estresse e melhora do bem estar. Funcionou como uma ferramenta de interação entre cuidador-criança-equipe, por meio do diálogo sobre as diferentes formas de se estimular o desenvolvimento infantil, respeitando as condições clínicas da criança e a habilidade do cuidador quanto ao cuidar.

Durante o processo, foi analisada a devolutiva dada pelos cuidadores, facilitadores e membros da equipe. Os tópicos identificados foram relacionados a necessidade de quebra do ócio hospitalar, ao envolvimento dos cuidadores e a influência da atividade na humanização da enfermaria, gerando uma mudança na postura dos cuidadores e melhora das relações interpessoais entre cuidador-criança-equipe.

\section{Referências}

Brasil (2017) Ministério da Educação. Base nacional curricular. Brasília.

Brasil. (2020). Ministério da Saúde. Caderneta da criança. Passaporte para a cidadania. Brasília.

Bustamante, V., Neves, D., Matos, M. S. D., \& Oliveira R. S. (2014) O brincar em família como possibilidade de humanização para crianças no hospital. Rev. EPOS, 5(2), 293-310.

Chesani, F. H. Andrade, M. G. C. \& Kerkoski, E. (2019). Vulnerabilidade de empatia de cuidadores/familiares de pacientes hospitalizados como sentimento reflexo a equipe assistêncial. Revista Univap, 25(48), 149-159.

Dahdah, D. F., Carvalho, A. M. P., Delsim, F. C., Gomes, B. R., \& Miguel, V. S. (2013). Grupo de familiares acompanhantes de pacientes hospitalizados: estratégia de intervenção da Terapia Ocupacional em um hospital geral. Cad. Ter. Ocup. UFSCar, 221(2), $399-404$.

Joaquim, R. H. V. T., Silvestrini, M. S., \& Marini, B. P. R. (2014). Grupo de mães de bebês prematuros hospitalizados: experiências de intervenção de Terapia Ocupacional no contexto hospitalar. Cad. Ter. Ocup. UFSCar. 22(1), 145-150.

Menezes, A. H. N., Duarte, F. R., Carvalho, L. R., \& Souza, T. E. S. (2019). Metodologia Científica Teoria e Aplicação na Educação a Distância. Petrolina. Fundação Universidade Federal do Vale do São Francisco.

Minayo, M. C. S., Deslandes, S. F., \& Gomes, R. C. (2011). Pesquisa Social: teoria, método e criatividade (Temas Sociais). (34a ed.). Vozes.

Moura, M. M. D., Guimarães, M. B. L., \& Luz, M. (2013) Tocar: atenção ao vínculo no ambiente hospitalar. Interface. 17(45), 393-404. .

Pereira, A. R. P. F., Matsue, R. Y., Vieira, L. J. E. S., \& Pereira, R. V. S. (2014). Análise do cuidado a partir das experiências das mães de crianças com paralisia cerebral. Saúde Soc. 23(2), 616-625.

Pereira, A. S., Shitsuka, D. M., Parreira, F. J., \& Shitsuka, R. (2018). Metodologia da Pesquisa Científica. Santa Maria, Brasil: Santa Catarina.

Pinheiro, I. S., Guerra, B. C. O., Silva, L. R., Pacheco, P. Q. C., Monnerat, I. C., Teixeira, S. V. B., Macedo, E. C., \& Veras, R. C. (2021). Impacto na saúde da mulher mãe cuidadora da criança com câncer. Research, Society and Development. 10(1), e31510111828.

Pyló, R. M., Peixoto, M. G., \& Bueno, K. M. P. (2015). O cuidador no contexto da hospitalização de crianças e adolescentes. Cad. Ter. Ocup. UFSCar. 23(4), $855-862$. 
Research, Society and Development, v. 10, n. 3, e39610313174, 2021

(CC BY 4.0) | ISSN 2525-3409 | DOI: http://dx.doi.org/10.33448/rsd-v10i3.13174

Ribeiro, W. A., Coutinho, V. V. A., Eduardo, C. R. B., Silva Neto, P. F., Pereira, B. V. O., \& Costa, V. S. (2020). Contributos do brinquedo terapêutico no processo de cuidado a criança $\quad$ hospitalizada: um estudo da literatura. Research, Society and Development. 9(7), e1000974706.

Rodrigues, A. A., \& Albuquerque, V. B. (2019). O brincar e o cuidar: O olhar da Terapia Ocupacional sobre o comportamento lúdico de crianças em internação prolongada. Interinstitutional Brasilian Journal of Occupational Therapy. 4 1), 27-42.

Silva, F. R., Ballarin, M. L. G. S., \& Oliveira, J. C. Grupo de acompanhantes de crianças internadas em unidade de terapia intensiva. Cad. Ter. Ocup. UFSCar. 2015; 23(4):871-877.

Souza, D. S., Figueiredo, B. A., \& Silva, A. C. D. (2017). O brincar de crianças com deficiência física sob a perspectiva dos pais/The play of children with disabilities from the perspective of parentes. Cadernos Brasileiros de Terapia Ocupacional. 25(2), 267-274.

Sposito, A. M. P., Garcia- Schinzari, N. R., Mitre, R. M. A., Pfeifer, L. I., Lima, R. A. G., \& Nascimento, L. C. (2018). O melhor da hospitalização: contribuições do brincar para o enfretamento da quimioterapia. Artículo de Investigación. 36(3), 328-337.

West, M. G. L. N., Vasconcelos, M. G. L., Coriolano- Marinus, M. W. L., \& Araújo, E. C. (2020). Care demands experienced by family caregivers of children with Primary Immunodeficiency. Revista Brasileira de Enfermagem. 73(4), e20180795. 